\title{
Satellite-delivered continuing medical education in Europe
}

\author{
James G Geraghty, Howard L Young
}

\begin{abstract}
Summary
There is increasing recognition of the need for continuing medical education in the medical profession. There are now many ways of delivering medical education including conferences, books, journals amongst others. This paper describes a novel method of delivering medical education using satellite transmission. This medium allows live medical education programmes to be broadcast to over 150 receiver sites in Europe. It also enables two-way live satellite links to be made between countries during the broadcast. EuroTransMed has an editorial board, in much the same way as a journal, which is representative of the differing medical societies in Europe. As the barriers between the various countries fall, EuroTransMed is an ideal medium to promote high quality, easily accessible, continuing medical education at a pan-European level.
\end{abstract}

Keywords: continuing medical education, satellite transmission, EuroTransMed

General Surgical Oncology, European Institute of Oncology, via Ripamonti 435, 20141 Milan, Italy JG Geraghty

Postgraduate Studies, University of Wales College of Medicine, Heath Park, Cardiff CF4 4XN, Wales, UK HL Young

Accepted 16 October 1995
Continuing medical education (CME) is a key component of professional medical development. The introduction of minimal invasive surgery and the impact of molecular biology are testimony to the need for continual updating of information. Traditionally this has been achieved by medical conferences, group learning and utilisation of a wide source of literature from libraries. It is well recognised, however, that these $\mathrm{CME}$ activities may be time consuming and do not easily address the needs of the individual. In recent years there has been an energetic approach to the provision of medical education in a more user-friendly manner. This has been fuelled by recent advances in technology.

\section{The evolution of EuroTransMed}

The provision of medical education by satellite is an attractive alternative to conferences and other standard CME activities. The transmission of live events such as news and sport by satellite is well established and popular among the general public. Despite a model of effective distribution of information and established audiovisual technology, live transmission for CME by satellite has been comparatively slow in development. This may be attributed to problems of marrying together the medical profession and the expertise required for satellite narrowcast transmission.

In 1988 the European Space Agency identified the possibility of an experiment for medical education using their Olympus I satellite. An approach was made to interested medical educators who put together a pilot experiment entitled EuroTransMed. Initially with funding from the Dutch Ministry of Education and the University of Leiden, this project met with a number of technical problems related to the satellite itself. Throughout this initial period the philosophy was that EuroTransMed should be non-profit-making and nonpromotional. As the problems with the Olympus Satellite continued it was decided that EuroTransMed should enter the commercial arena providing high quality programmes which maintained integrity and were non-promotional. Having found a sponsor, a limited series of programmes was successfully broadcast to evaluate the feasibility of the medium for medical education purposes.

\section{Structure of EuroTransMed}

From this initial phase it was decided to formalise and standardise the delivery of medical education by satellite for Europe within the concept of EuroTransMed. An Editorial Board was established similar to that of a journal. It consisted of a chairperson with constituents drawn from countries in Europe to maintain its pan-European philosophy. Representation from major European societies including the European School of Oncology, the European Atherosclerosis Society and the European Organisation for Research and Treatment of Cancer have also joined the Editorial Board, which meets twice yearly with a remit to decide on the future expansion of the project as well as the format and scientific content of programmes. This information is then passed to a Medical Editor for treatment and development of programmes in collaboration with a production company. The Board receives submissions of possible programmes and subject areas and these are also considered and may be subject to a refereeing process similar to that of journals as EuroTransMed expands.

How can EuroTransMed provide high quality programmes technically while maintaining scientific content? Organisationally this has been achieved by having a professional broadcast production team, managed by an executive producer, who interrelate with the Editorial Board. Included within the production team is the dedicated Medical Editor who forms the bridge between the Editorial Board and the nature of the scientific output with the technical aspects of high quality programme production. 


\section{Programme structure}

The live transmission of a programme related to a medical topic is the model most representative of the aims of EuroTransMed. Each programme consists of up to four people, three speakers and a chairperson, who are recognised experts in their field. Preparation is important as many of the speakers do not have experience in delivering lectures on live television. A formal dress rehearsal takes place prior to the live broadcast. The method of delivery of medical knowledge is similar to that of medical conferences. Slides, illustrations and video can be interspersed during each presentation. A strict time limit of seven to nine minutes enables a panel discussion to take place between participants.

The interactive component of any educational exercise is a key source of productivity for the interchange of ideas. A unique feature of each EuroTransMed programme is that, following the panel discussion, interaction takes place by fax or telephone, whilst more recently live communication between the panel and receiver sites has been undertaken using videophone/ISDN and occasionally two-way satellite links. The use of such communication technology is one of the distinguishing features of EuroTransMed, allowing it to have live interactive discussion across Europe.

The programme content is variable, covering a range of subjects (box 1 ).

EuroTransMed programmes for April 1996

2 April: to be announced 16 April: Management of progressive renal disease

23 April: to be announced 30 April: Anti-retroviral therapy options for HIV infection All programmes are shown at $1300 \mathrm{~h}$ Central European time
Programmes are targeted to a wide audience of differing specialties allowing a broad-based exposure of EuroTransMed to the medical profession. The content of future programmes depends on feedback received from previous programmes. A Network Manager based in Leiden (The Netherlands) liaises and visits sites in Europe on a regular basis to audit the response at a local level. This is supplemented with the use of a reply-paid questionnaire which can be returned following each programme. Programmes are held weekly during the academic term with 35 programmes broadcast in 1992/93 and 36 in 1993/94.

\section{End users}

The distribution of receiver sites is shown in the table. A total of 210 sites in 21 countries in Europe were projected for December 1995. Broadcasts take place at 1300 hours Central European time which was chosen to coincide with the lunch hour. However, it has been acknowledged that the timing of programmes is difficult since it has not been possible to identify a first-choice time for all disciplines in all the various countries served by EuroTransMed. For instance,

Table EuroTransMed receiver sites by country

\begin{tabular}{|c|c|c|}
\hline Country & $\begin{array}{l}\text { August } 1995 \\
\text { (installed) }\end{array}$ & $\begin{array}{l}\text { December } 1995 \\
\text { (projected) }\end{array}$ \\
\hline Austria & 1 & 2 \\
\hline Belgium & 7 & 19 \\
\hline Croatia & 1 & 1 \\
\hline Czech Republic & 9 & 10 \\
\hline Denmark & 3 & 5 \\
\hline Finland & 3 & 4 \\
\hline France & 4 & 9 \\
\hline Germany & 6 & 18 \\
\hline Greece - & 1 & 2 \\
\hline Hungary & 11 & 12 \\
\hline Ireland & 2 & 4 \\
\hline Italy & 6 & 8 \\
\hline Lebanon & 1 & 1 \\
\hline Macedonia & 0 & 2 \\
\hline Norway & 1 & 2 \\
\hline Poland & 14 & 14 \\
\hline Portugal & 2 & 2 \\
\hline Romania & 1 & 2 \\
\hline Slovakia & 4 & 5 \\
\hline Spain & 6 & 9 \\
\hline Sweden & 1 & 2 \\
\hline Switzerland & 3 & 5 \\
\hline The Netherlands & 16 & 20 \\
\hline Tunisia & 1 & 1 \\
\hline United Kingdom & 46 & 51 \\
\hline Total & 150 & 210 \\
\hline
\end{tabular}

Potential reach from existing satellite (Turkey, Lebanon, Israel, Northern Egypt, North Africa) 


\begin{tabular}{|l|}
\hline Key features for \\
EuroTransMed \\
\hline - delivery of CME by satellite \\
- live television broadcast \\
- two-way live link between countries \\
- editorial board \\
- accreditation for CME approved \\
\hline
\end{tabular}

Box 2 surgeons operating until late afternoon will miss lunchtime broadcasts. It is sometimes necessary therefore to delay the broadcast if it is on a surgical topic to a more appropriate time. Alternatively, recordings of the live lunch-time transmission have been replayed in the late afternoon to enable those who have been unable to participate in the live interactive component to watch aspects of the programme pertinent to their own needs.

The other major educational advantage of EuroTransMed is that videos of each programme can be used in teaching environments. The delivery of recognised experts in their field in video format can stimulate local discussion. A library of over 100 such videos already exists.

\section{Future developments}

EuroTransMed has the ability by the nature of the footprint of the satellite to reach doctors throughout Western and Central Europe. The target audience varies according to the subject matter which is broadcast, but it enables experts in specific areas to be seen by doctors in their own environment and at the same time to interact with those individuals. EuroTransMed is currently discussing utilisation of the HISPASAT Satellite to enable programmes to reach South and Latin America. Implicit in this project is the need for simultaneous translation into the relevant language and this is an area which is currently under evaluation.

More practically, but equally important, is obtaining formal recognition from the European Community of the importance of EuroTransMed as a medium for delivering CME. The initial development of EuroTransMed sites in Central Europe was funded under the EC TEMPUS programme and more recently an application to the Telematics part of the 4th Framework of the European Commission was successful in the field of oncology. This will enable a small number of programmes to be produced specifically in the area of oncology and broadcast throughout Europe looking to achieve dissemination of information to medical practitioners.

As EuroTransMed develops and as CME becomes a key component in the continuing professional development it will be essential that the programming conforms to the requirements of accreditation. No standardised CME credit system exists in Europe at present. However, the Editorial Board of EuroTransMed has already received accreditation from the Royal College of Physicians with the proviso that a nominated local organiser acts as a moderator to promote discussion and verify attendance. This approval applies equally to other Royal Colleges in the UK. Similarly, the Dutch Society of Cardiology has approved EuroTransMed programmes in cardiology in a similar manner, for physicians in The Netherlands. As Europe moves towards a more formal system of CME accreditation, EuroTransMed will aim to work with relevant societies and awarding bodies to fulfil the requirements needed for external credits for physicians and doctors throughout Europe.

\section{Conclusion}

The delivery of medical information is vital for continuing medical excellence. This is achieved by interaction between individuals at an international as well as national level. Often this is limited because of distance and the need for cost effectiveness. Satellite-delivered CME to Europe overcomes this limitation and has the potential not only to facilitate the educational process, but also to increase substantially the numbers of physicians participating in CME programmes. 